\title{
90 UNBIASED PROTEOMIC PROFILING LEADS TO THE DISCOVERY OF A NOVEL NON-INVASIVE BLOOD-BASED PROTEIN PANEL WITH SIGNIFICANT POSITIVE PREDICTIVE VALUE IN PANCREATIC AND COLORECTAL CANCERS
}

Kristina Beeler*, Roland Bruderer, Marco Tognetti, Kamil Sklodowski, Sebastian Mueller, Dominique Kamber, Lukas Reiter. Biognosys, Schlieren, Switzerland

Background Mass spectrometry-based discovery proteomics has recently emerged as a high-throughput method for the proteomic profiling in biofluid samples from large clinical and population screening cohorts. Despite this progress, a significant fraction of the plasma proteome is currently not covered by state-of-the-art discovery approaches and therefore not accessible for biomarker discovery. To close this analytical gap, we present a novel workflow combining automated plasma depletion and FAIMS-DIA-MS to bridge both sensitivity and scalability. We demonstrate the applicability of this workflow to support biomarker discovery and subject stratification in precision oncology in a case-control cohort.

Methods The plasma samples were depleted in 96-well format using an automated MARS-14 depletion system. The depleted samples were processed to tryptic peptides and analyzed using a Thermo Scientific Orbitrap Exploris 480 equipped with a FAIMS Pro device. Data processing and analysis were performed using Biognosys' SpectroMine and Spectronaut software.

Results Using the unbiased discovery workflow, we investigated a cohort comprising of 180 plasma samples from healthy donors and subjects diagnosed with pancreatic, breast, prostate, colorectal and lung (NSCLC) cancer at either early or late stage of the disease. Overall, the optimized FAIMS-DIAMS quantified 2,741 proteins across all samples and 1,849 proteins on average per sample measurement. Based on estimated plasma protein concentrations (Human Protein Atlas), quantified proteins span across 8 orders of magnitude, down to single digit $\mathrm{pg} / \mathrm{mL}$. Within this dynamic range, we could interrogate the tissue leakage proteome, interleukins and signaling proteins. Using classification algorithms, we were able to select candidates to build protein panels which provide significant positive predictive values associated with different disease stages, especially in the sub-cohorts for pancreatic and colorectal cancer.

Conclusions We demonstrate the capabilities of a novel discovery workflow for deep, quantitative profiling of plasma samples at large scale, providing a rich proteomic resource for precision oncology.

http://dx.doi.org/10.1136/jitc-2021-SITC2021.090 